\title{
Evaluation of Various Hand Controllers for Use by a Space Suited Subject
}

\author{
Lauren S. Shook and David L. Akin \\ Space Systems Laboratory, University of Maryland at College Park
}

Copyright (@ 2001 Society of Automotive Engineers, Inc.

\begin{abstract}
The Space Systems Lab has evaluated several different types of generic hand controllers to see which performs the best when used by a suited subject. This paper outlines the types of hand controllers selected for this experiment and the results of the performance testing. The evaluation was conducted by subjects wearing spacesuit gloves in a partial pressure glovebox at a pressure differential of 4.3 pounds/square inch. Performance for each hand controller was measured by the completion of several one degree-of-freedom (DOF) tasks presented to the subject on a computer screen. Performance metrics for this experiment included the error associated with attempting to follow an ideal trajectory and a subjective Cooper-Harper questionnaire given after each session was complete. The same information was also collected for unpressurized suit gloves and for the bare hand.
\end{abstract}

\section{INTRODUCTION}

The goals of spaceflight today are somewhat different than our earlier goals. Originally, our goals were to simply prove that we could reach the heights we were aiming for; each step simply took us higher than before. Today, we've graduated to research goals on-orbit and have begun looking toward manned bases on both the moon and Mars.

With the advent of the International Space Station (ISS), the number of hours spent on-orbit and involved with extravehicular activity (EVA) will dramatically increase. Just the number of hours slated for the construction of the ISS will more than double the total number of hours of EVA in the previous US history of spaceflight [1]. This increase has become known as the "EVA Wall."

This unprecedented increase poses many challenges to the EVA community. Among these are to make the most of limited resources, to make astronauts more efficient in completing tasks, and to provide ease of movement without sacrificing safety.
The goals of this research included examining input devices to evaluate their appropriateness for use by suited subjects. In doing so, it was hoped that this might further illuminate how tasks are accomplished while wearing gloves and what types of movement are the most accurate and least fatiguing. This information could be used in the future to design input devices for specific tasks or even in the design of new gloves.

\section{HISTORY}

Our hands are not just another part of our anatomy; they are our tools and our means of expression. We continuously use them to interact with our environment and shape it to our needs. Consequently, the need for gloves to protect our hands in space has also hindered our interaction with that environment.

Gloves, in general, account for a decrease in dexterity and tactility. Early studies using gloves of leather or wool showed that "the efficiency with which instrument controls may be operated by a gloved hand depends on certain measurable glove characteristics" [2]. Bradley reported that previous comparisons between barehanded and gloved manipulability showed a marked difference between the two; for all six types of gloves tested, performance was significantly lower than barehanded performance [3].

Space suit gloves are comprised of several layers of material, including a pressure bladder, a restraint layer, and a thermal micrometeorite layer. Also, fingertips are coated in rubber to help increase friction. While protecting the hand well, these layers prohibit the suited subject from being able to accurately sense the objects that they are manipulating.

In addition, space suit gloves do not maintain a constant volume. Motions by the hands and fingers tend to change the volume. This, coupled with the pressurization of the suit, leads to the gloves having a preferred position. The subject then has to constantly fight against that preferred position when trying to accomplish a task. This type of 
compensation can lead to muscle fatigue, especially in the fingers and wrist [4].

In the next hundred years of space exploration, we are looking toward larger scale missions and more complicated tasks than ever before in our history. Future exploration of both the Moon and Mars will require a great deal of planning and development of new technology in order to be carried out successfully. Given the success of smaller robots, such as Sojourner, on Mars, it seems only logical that robots would play a role in future planetary exploration missions. It has been proposed [5], [6], [7] that a larger robot would be useful as an astronaut assistant for planetary exploration. Such a robot would be capable of carrying scientific equipment, storing samples, and carrying consumables for the astronaut. It could also serve as a terrain scout or take video of an interesting area.

Field testing has shown that it would be advantageous that the "EVA crewmember be provided with some control of the rover" and future study was recommended to develop "methods for the suited subject to control the robotic rover" [8].

Gloves hold their own unique limitations, such as limited range of motion and dexterity. These impact the performance of hand controllers designed for nude body performance. To this end, a control method appropriate to a suited subject would need to be found.

This experiment was designed to illustrate which basic type of hand controller would be the most appropriate for use by a suited subject. Also, it is hoped that the results may provide insight into designing hand controllers for future missions, both for EVA and planetary surface exploration. In addition, these experiments may provide insight into the performance of space suit gloves.

\section{EXPERIMENT DESIGN}

\section{DESIGN GOALS}

This experiment uses four separate one degree-offreedom (DOF) tasks displayed on a computer to test the performance of several different hand controllers. Each subject completed all four tasks under three different conditions: no glove, unpressurized glove, and pressurized glove. The details of these input devices and the tasks that were presented are outlined in the remainder of this section.

\section{HAND CONTROLLER TYPES}

Four different hand controller types were selected for this experiment. The selection criteria and the types selected are discussed below. The goal behind these choices was to represent both current styles of input devices and new and innovative methods.

\section{SELECTION CRITERIA}

A working definition of prehension has been stated as "the application of functionally effective forces by the hand to an object for a task, given numerous constraints" [9]. The hand has the ability to move in many different ways to complete tasks, but some movements and hand shapes are more effective than others. In completing a task, three demands are placed upon the hand and its posture:

1. Apply forces to match the anticipated forces in the task (stable grasp)

2. Impart motion to the object (manipulate) or transport the object as necessary

3. Gather sensory information about the state of interaction with the object during the task in order to ensure grasping and manipulative stability [10].

Many postures of the hand have been catalogued over the years. A simple set of hand postures was developed by G. Schlesinger in 1919 in order to categorize the functionality of prosthetic hands [11]. The basic set of six postures include cylindrical, fingertip, hook, palmar, spherical, and lateral.

The goal of this selection was to choose each controller to be representative of a basic type of hand motion. In this way, it could be seen how well the types of hand motions could be used to complete tasks while wearing a suit glove. All of the devices selected were commercial off-the-shelf devices, designed for barehanded use. It was hoped that a clear differentiation would be seen between the four input devices under the three gloved conditions, indicating which type of motion might be more appropriate for use by a gloved subject.

\section{TYPES SELECTED}

The following hand controller types were selected for this experiment: Mouse, Touchpad, Joystick, and Magnetic motion sensor. They can be seen in Figure 1.

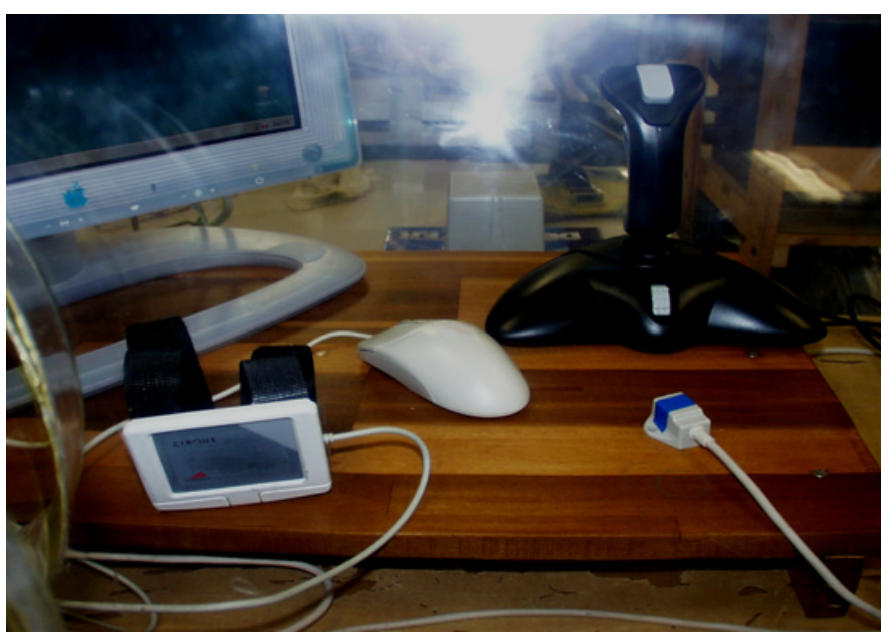

Figure 1: Hand Controllers Selected from $L$ to $R$ : Touchpad, Mouse, Joystick, Magnetic Motion Sensor. 
The mouse was selected as a typical input device that all subjects would be familiar with. The motion involved is a side to side motion in a single plane. Using this device also requires the subject to be able to hold the input device in a spherical grip and control its motion. While it is clear that the mouse would not be suitable as an operational input device, it provides a useful comparison point for the other controllers.

\section{Joystick}

The joystick chosen here was an Advanced Gravis (PC), Gravis Destroyer PC Joystick. This is a standard three DOF PC joystick. A fourth DOF can be enabled using a wheel on the base of the joystick, but was not enabled for this experiment. This hand controller requires that the subject be able to grip the handle in a cylindrical posture and move about the neutral zero position.

\section{Touchpad}

A touchpad is an input device that uses an interruption of the conductive surface to recognize the user's position on the surface of the pad. Touchpads are typically a "single plastic mylar membrane silkscreened with a silver conductive grid and two resistive strips." [12] When touched, the $X$ and $Y$ resistances are measured and the position is calculated. The body typically provides the conductive surface that registers on the touchpad's surface. For the gloved test cases, a ground wire was required to be attached to the glove's fingertip in order to use this input device. The touchpad chosen for this experiment was a Cirque Easy Cat Touchpad, which can be seen in Figure 1.

Touchpads or advanced LCD displays have long been considered candidates for controllers on EVA missions. The NASA EVA Research and Technology Roadmap of September 1999 shows interest in developing a "small lightweight cuff mounted or visor integrated display system." [13] In this vein, the touchpad was placed on the back of the left hand using Velcro loops. The subject was then able to use a fingertip from the right hand to control the touchpad.

\section{Magnetic motion sensor}

Flock of Birds is a motion sensing package designed by Ascension Technology Corporation. It is capable of tracking up to 4 sensors simultaneously. However, for this experiment only one sensor was needed. Each sensor position is measured relative to the fixed transmitting antenna, which is driven by a pulsed, direct current (DC) signal. The receiving antenna measures both the transmitted pulse and the earth's magnetic field. A microprocessor controls both the transmission and receiving sides, as well as processing the received signal into position and orientation data. [14] The system can be seen in Figure 1.
The magnetic position sensor was placed on the back of the right hand and held in place using a Velcro strip. In this case, the motion involved is of the arm to position the hand.

\section{TASK SELECTION}

The tasks chosen for this experiment were designed to be simple, so that the response could be studied in only one degree of freedom. It was thought that a one DOF task would be similar to that of adjusting a heading angle or increasing/decreasing speed; tasks that might be applicable to controlling an astronaut assistant rover.

The following sections will discuss the four one DOF tasks chosen, the theory behind them, and the performance metrics used to evaluate the results.

\section{Tracking Tasks}

Typically, human performance is measured using an "information processing approach." [15] These are tasks where a stimulus is presented to the subject and the result of the reaction to that stimulus can be measured. A type of continuous test used by many researchers is a tracking test. In a tracking test, the subject is presented with an ideal case and asked to mimic it as best possible, keeping the error to a minimum.

The four tracking tests used for this experiment can be classified into two types, pursuit and compensatory. Three pursuit tasks were chosen with the following ideal trajectories: a sine wave, a sine sweep, and a step. The fourth task was a compensatory tracking task using a sine wave as the target path. These tasks are discussed in more detail below.

\section{Pursuit Tracking Tasks}

Pursuit tracking tasks are defined as those in which "the instantaneous reference input $r$ and the instantaneous controlled input $y$ are both displayed to the human operator separately and independently, so that he may distinguish individual properties of these signals by direct observation." [16] In this case, two icons representing the reference input, or target, and the controlled input were displayed to the subject. As seen in Figure 2, the target icon was displayed on screen above the controlled icon, to remove any confusion should the objects overlap. Subjects were asked to follow the target icon as closely as possible using the controlled icon.

The first pursuit task was following a simple sine wave, fixed in amplitude. The reason for choosing this tracking task was to examine the mean error for a simple, unchanging task. The second task was following a sine wave sweep. In this case, the amplitude of the sine wave remained fixed while the frequency increases during the test. It was hoped to gain both mean error and frequency response data from this task. Lastly, a step pursuit task 
was chosen. In this case, the target icon begins at zero, and then jumps to a set distance on the screen. From this test, it was hoped to collect data on settling time, overshoot, and error.

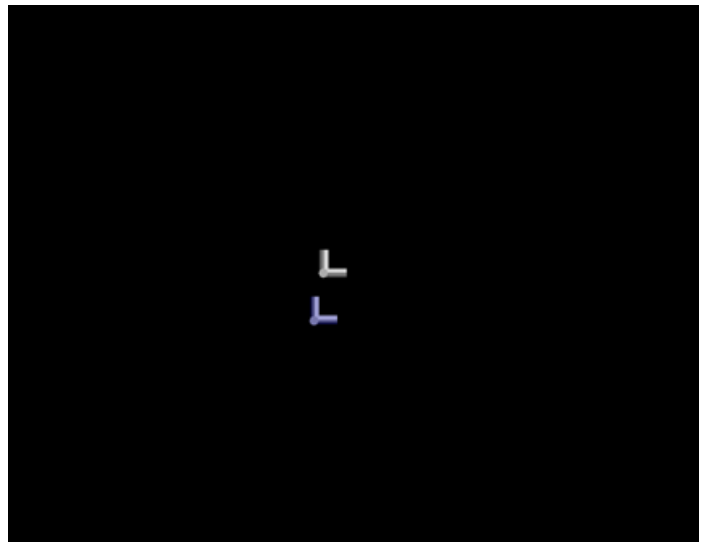

Figure 2: Controlled and Target Icons

\section{Compensatory Tracking Task}

A second type of tracking task is called compensatory tracking. This is defined as "one in which the human operator has a single input, the error $e$, the difference between actual system response $y$ and ideal response (the reference input)." [17]

For this experiment, the subjects only viewed an icon representing the error present in the test and a vertical line on the screen at zero. This can be seen in Figure 3. They attempted to keep the icon on the line, using the various hand controllers. The reference input for this case was unknown to the subjects and was a simple sine wave, identical to the first pursuit tracking task.

This task is considered to be advantageous, since inputoutput characteristics of the system can be measured directly. This is because the human operator is assumed to be a function of one variable, the error, instead of two, the input and the output. [18]

\section{Performance Metrics}

The performance metrics for this experiment include both analytical and subjective. As stated above, the mean error involved in task completion was calculated for all tracking tasks and compared between hand controllers. It was hoped that this would give a clear indication of which hand controller might be appropriate for use by a gloved subject.

The sine sweep task was examined for the frequency response of the subject, as well as for mean error. For the step task, settling time and overshoot were examined.

Subjective data was taken using a modified Cooper Harper rating scale for each hand controller under each glove condition. Subject comments on tactility, dexterity, and fatigue were also recorded.

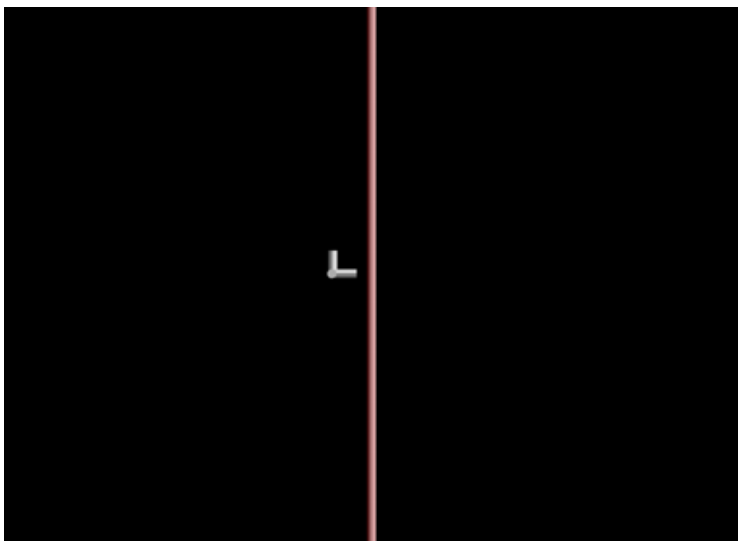

Figure 3: Compensatory Tracking Task

The results given in this paper will be limited to the mean error for the sine, sine sweep and compensatory tracking results and the Cooper-Harper subject ratings. A more detailed description of the experiment, complete results, and subject comments can be found in [19].

\section{EXPERIMENTAL SETUP}

All subject testing was conducted at the Space Systems Laboratory, at the University of Maryland. A differential pressure glovebox, on loan from NASA Goddard Spaceflight Center, was used for all of the tests. In both the barehanded and unpressurized glove cases, the subject completed the tests in an open, unpressurized glovebox. This was done to maintain the same constraints on arm position as the pressurized glove tests. The glovebox used is constructed of Plexiglas and operates at a 4.3 PSI pressure differential.

In order to use the hand controllers in the pressurized glovebox, a new end plate needed to be designed. The plate was designed to fit the previous interface and included connectors for all of the hand controllers.

\section{LAYOUT}

The glovebox was set up in the following way for all tests. A flat panel monitor was placed inside the glovebox, to the left of the subject, on a low table. The subject could change both the position and the orientation of the monitor. Each input device was positioned by the subject to where it was comfortable and operated the easiest. Figure 4 shows the glovebox with monitor and input devices. Subjects were permitted to either stand or sit while completing each series of tests. 


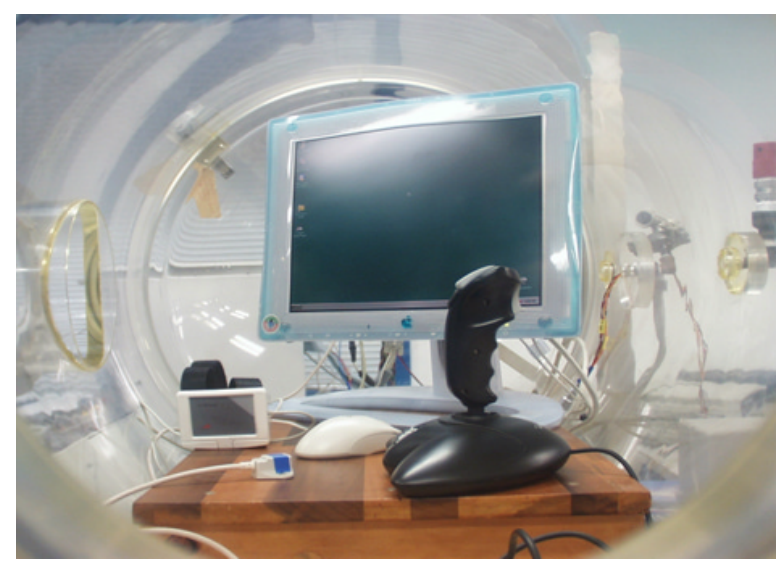

Figure 4: Glovebox Setup

\section{SOFTWARE}

The software for this experiment was written in C, using Open GL, GL utilities (GLUT), and a graphics package called World Modeler. A separate program was written for each input device. Each program recorded subject number, test number, test condition (bare-handed, unpressurized glove, pressurized glove), and trial number. Based on these inputs, the proper task was displayed on the screen for the subject. The data file for each completed task included time, actual position, desired position, and position error.

\section{GLOVES}

Two gloves were used for this experiment. The righthanded glove was a Shuttle suit glove, series 3000 , on loan from ILC Dover. It can be seen in Figure 5. This was not a standard glove, however, and used an experimental Thermal Micrometeorite Garment (TMG) not found on standard 3000 series gloves. Any results contained herein do not accurately reflect the performance of any specific type of glove, but are simply meant to illuminate the general performance of pressurized gloves.

The left glove used was a Skylab suit glove. The connection ring was redesigned to fit the wrist connection of a shuttle glovebox arm segment. This glove, as seen in Figure 5, was not used to control any of the hand controllers. Its purpose was to allow the use of the touchpad on the back of the left hand under pressurized conditions.

Both gloves were connected to Shuttle arm segments. The glove and segments were then placed in the glove ports of the glovebox.

All subjects selected were right-handed; this was deemed best to utilize the superior features of the more modern glove. Since only one set of gloves was available for testing, it was not practical to attempt to limit test subjects to those with ideal fits to the gloves. In order to assess the impacts of this limitation, hand measurements were taken of each subject, along with measurements of the gloves used.
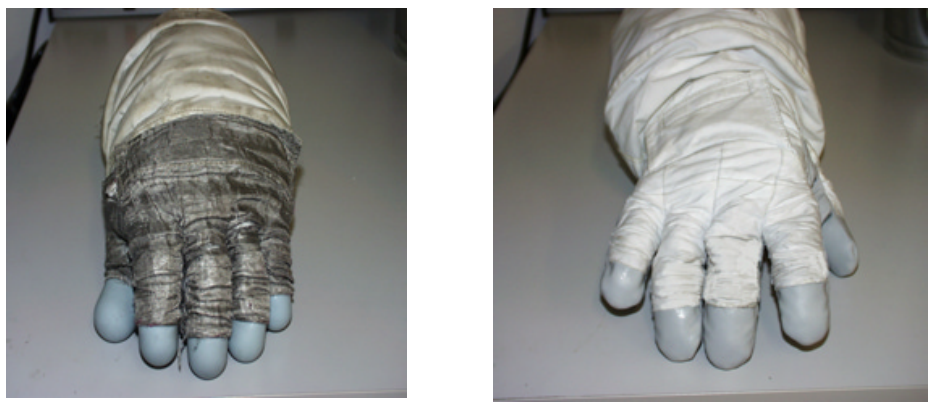

Figure 5: Left and Right Gloves

\section{TEST PROCEDURE}

The test matrix was comprised of four input devices, three glove conditions, four tasks to be completed, and five trials for each. Therefore, each input device required 60 trials. This resulted in a total of 240 trials for each subject and 1440 trials for the whole experiment. These were broken up into four sessions, one for each input device. Each session lasted approximately an hour and half in duration.

Prior to the start of testing, the entire testing procedure was explained to each subject and they were asked to fill out an informed consent form and a pre-test questionnaire. Subjects typically completed each series of trials for a single input device in an hour and a half.

For each input device, each subject began with barehanded testing. They began with test 0 , the sine wave, and completed five 30 second trials. Next, they completed five 30 second trials of the sine sweep, test 1 . Third was five trials of test 2 , the step, which took approximately $15-20$ seconds to complete. Lastly, they completed five 30 second trials of the compensatory tracking test. This same sequence was then repeated with the unpressurized glove, and then the pressurized glove.

After the sequence was completed for each glove condition, the subject filled out a post-test questionnaire and gave the input device a Cooper-Harper rating.

The procedure above was followed for all input devices. All subjects began with the mouse, but there was no specific order for the other three devices. Total subject testing time was approximately six hours.

\section{RESULTS}

\section{MEAN ERROR RESULTS}

The mean error was taken for each trial. Then all of the trials for all of the subjects were complied for each task type. The matrix for each task type consisted of 360 data points. An Analysis of Variance (ANOVA) was run for 
each task using the SAS program, as was a Duncan range grouping.

\section{SINE WAVE}

Looking at the ANOVA results for the sine wave task, the subject, glove condition, and hand controller type are all statistically significant to the .0001 level. The trial number was not found to be statistically significant to the .001 level for this task.

\begin{tabular}{|c|c|}
\hline Source & Pr>F \\
\hline Subject & .0001 \\
\hline Glove Condition & .0001 \\
\hline Hand Controller & .0001 \\
\hline Trial Number & .0837 \\
\hline
\end{tabular}

\section{Table 1: ANOVA Results for Sine Wave}

The Duncan range grouping shows that subjects are clearly grouped by gender.

\begin{tabular}{|l|l|l|}
\hline Duncan Grouping & Mean & Subject Number \\
\hline A & 1.10622 & 2 \\
\hline A & .96319 & 1 \\
\hline A & .95599 & 6 \\
\hline B & .70265 & 3 \\
\hline B & .67186 & 5 \\
\hline B & .65925 & 4 \\
\hline
\end{tabular}

Table 2: Duncan Range Grouping by Subject for Sine Wave

Group A consists of subjects 1,2, and 6, which are all female. While group B consists of subjects 3,4 , and 5 , which are all male. Initially, this appears to be similar to the gender performance results which Bishu and Klute reported [20]. However, in reviewing the subject data, this appears to be less of a gender effect and more an artifact of the subject's hand size. Subject 4's hands were slightly too large for the glove and had reported the glove as being too tight during several tests. Subjects 1 and 2 both had similar hand sizes and both reported the glove as being too large and that they were unable to reach the ends of the fingertips.
As one might expect, the pressurized glove condition had the worst mean error for this task. The bare-handed and the unpressurized glove conditions are part of the same Duncan range grouping.

\begin{tabular}{|l|l|l|}
\hline Duncan Grouping & Mean & Glove Condition \\
\hline A & 1.01491 & Glove Pressurized \\
\hline B & .79592 & Glove Unpressurized \\
\hline B & .71875 & Bare Handed \\
\hline
\end{tabular}

Table 3: Duncan Range Grouping by Glove Condition for Sine Wave

The results of the hand controllers for the sine wave task are shown in Table 4. The magnetic motion sensor had the best performance, followed by the mouse, and then the grouping of the touchpad and joystick.

\begin{tabular}{|l|l|l|}
\hline Duncan Grouping & Mean & Hand Controller \\
\hline A & 1.06236 & Touchpad \\
\hline A & .98256 & Joystick \\
\hline B & .78086 & Mouse \\
\hline C & .54699 & $\begin{array}{l}\text { Magnetic motion } \\
\text { sensor }\end{array}$ \\
\hline
\end{tabular}

Table 4: Duncan Range Grouping by Hand Controller for Sine Wave

\section{Sine Sweep Task}

For the sine sweep task, the subject number, glove condition, and hand controller type were all found to be significant to the .0001 level. Again, the trial number was found to have no statistical significance.

\begin{tabular}{|l|l|}
\hline Source & Pr>F \\
\hline Subject & .0001 \\
\hline Glove Condition & .0001 \\
\hline Hand Controller & .0001 \\
\hline Trial Number & .9379 \\
\hline
\end{tabular}

Table 5: ANOVA Results for Sine Sweep 
The same gender groupings were also seen for this task, however subjects 2 and 3 were also grouped as a third group. The reason for this third grouping is unclear.

\begin{tabular}{|l|l|l|}
\hline Duncan Grouping & Mean & Subject Number \\
\hline A & 1.53396 & 1 \\
\hline A & 1.47185 & 6 \\
\hline A B & 1.34195 & 2 \\
\hline C B & 1.15550 & 3 \\
\hline C & 1.06336 & 5 \\
\hline C & 1.06218 & 4 \\
\hline
\end{tabular}

Table 7: Duncan Range Grouping by Subject for Sine Sweep

Again, the results show that the pressurized glove has the largest mean. The bare handed and unpressurized glove conditions have a smaller mean, with the bare handed being the smallest, and are grouped together, as seen in Table 8.

\begin{tabular}{|l|l|l|}
\hline Duncan Grouping & Mean & Glove Condition \\
\hline A & 1.46879 & Glove Pressurized \\
\hline B & 1.21456 & Glove Unpressurized \\
\hline B & 1.13105 & Bare Handed \\
\hline
\end{tabular}

Table 8: Duncan Range Grouping by Glove Condition for Sine Sweep

The results of the hand controllers for the sine sweep task were more interrelated than those of the sine wave. The magnetic motion sensor had the lowest mean, followed by the grouping of the mouse and joystick, and then the grouping of the joystick and the touchpad. This grouping indicates that the mouse and joystick are statistically similar and the joystick and touchpad are also statistically similar, but the mouse and touchpad are not related.

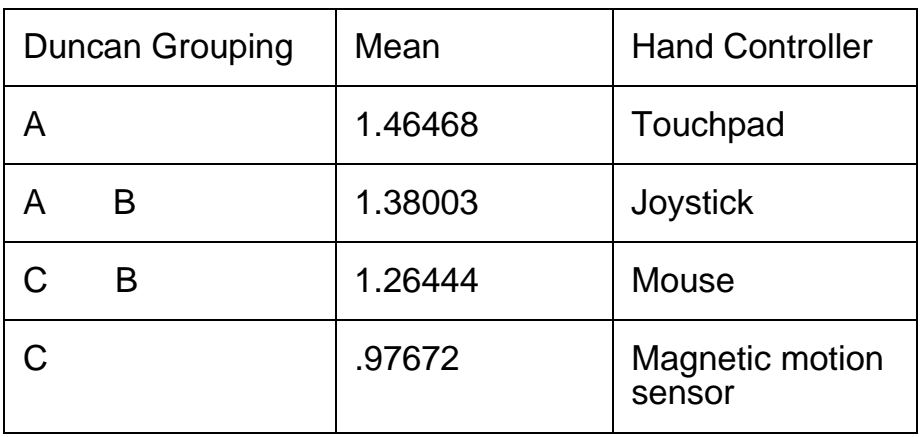

Table 9: Duncan Range Grouping by Hand Controller for Sine Sweep

\section{Compensatory Tracking Task}

The results from this task are different from those of the previous two tasks. The subject, glove condition, and hand controller type are all still statistically significant, but for this case the glove condition is now only significant to the .001 level (.0006) as opposed to the previous .0001 level.

\begin{tabular}{|l|l|}
\hline Source & Pr>F \\
\hline Subject & .0001 \\
\hline $\begin{array}{l}\text { Glove } \\
\text { Condition }\end{array}$ & .0006 \\
\hline $\begin{array}{l}\text { Hand } \\
\text { Controller }\end{array}$ & .0001 \\
\hline Trial Number & .4462 \\
\hline
\end{tabular}

Table 10: ANOVA Results for Compensatory Tracking Task

Also, for this task the subject grouping is no longer strictly by gender; Subjects $1,2,3$, and 6 are now grouped, 3,4, and 6 are grouped, and 5 is grouped separately.

\begin{tabular}{|c|c|c|}
\hline Duncan Grouping & Mean & Subject Number \\
\hline A & 1.34618 & 2 \\
\hline$A$ & 1.25622 & 1 \\
\hline A $B$ & 1.17907 & 3 \\
\hline A $\quad B$ & 1.15281 & 6 \\
\hline B & .98491 & 4 \\
\hline C & .72101 & 5 \\
\hline
\end{tabular}

Table 11: Duncan Range Grouping by Subject for Compensatory Tracking Task

Despite the glove condition being less significant, the results are still the same as in the previous two tasks. The pressurized glove has the highest mean and the bare handed and unpressurized glove conditions are still grouped.

\begin{tabular}{|l|l|l|}
\hline Duncan Grouping & Mean & Glove Condition \\
\hline A & 1.22398 & Glove Pressurized \\
\hline B & 1.07663 & Glove Unpressurized \\
\hline B & 1.01969 & Bare Handed \\
\hline
\end{tabular}

Table 12: Duncan Range Grouping by Glove Condition for Compensatory Tracking Task 
The hand controller results for the compensatory tracking task are as follows, the magnetic motion sensor has the lowest mean, then the mouse, the joystick, and finally the touchpad. The joystick and touchpad are grouped, while the magnetic motion sensor and mouse are in separate groups, as seen in Table 13.

\begin{tabular}{|l|l|l|}
\hline Duncan Grouping & Mean & Hand Controller \\
\hline A & 1.44145 & Touchpad \\
\hline A & 1.12959 & Joystick \\
\hline B & .95229 & Mouse \\
\hline C & .90374 & $\begin{array}{l}\text { Magnetic motion } \\
\text { sensor }\end{array}$ \\
\hline
\end{tabular}

Table 13: Duncan Range Grouping by Hand Controller for Compensatory Tracking Task

\section{COOPER-HARPER SUBJECT RATINGS}

The Cooper-Harper rating scale was originally designed to rate the flight handling qualities of aircraft by their pilots. It has evolved into a subjective method of evaluating human interaction with a system. This experiment used a modified version of the CooperHarper rating system, which can be found in [21], where a rating of 1 is excellent and 10 indicates major deficiencies.

Each subject gave the hand controller an overall rating, based on the tasks completed under all of the glove conditions. Figure 6 shows the ratings given for each hand controller under the barehanded condition.

Cooper-Harper Ratings for Hand Controllers - No Glove

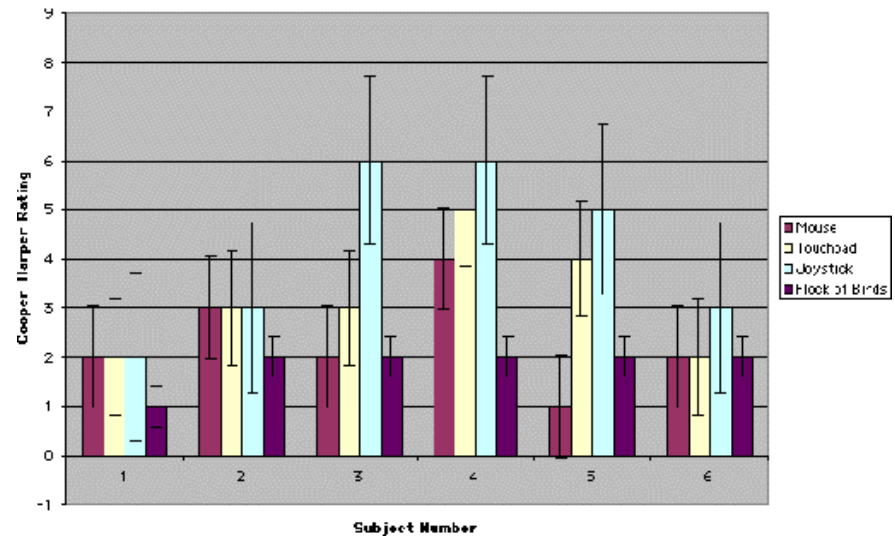

Figure 6: Modified Cooper-Harper Ratings For Hand Controllers Under Barehanded Conditions

As seen in the graph, the ratings vary by subject. Three agreed that the magnetic motion sensor did the best, while two tied it with other controllers. The mouse also did well; first with one subject and tied at first for two others. The joystick did badly as it had the highest rating for four subjects and was tied for the highest with the other two subjects. The touchpad ratings varied over all of the subjects.

Figure 7 shows the Cooper-Harper ratings for the unpressurized glove conditions.

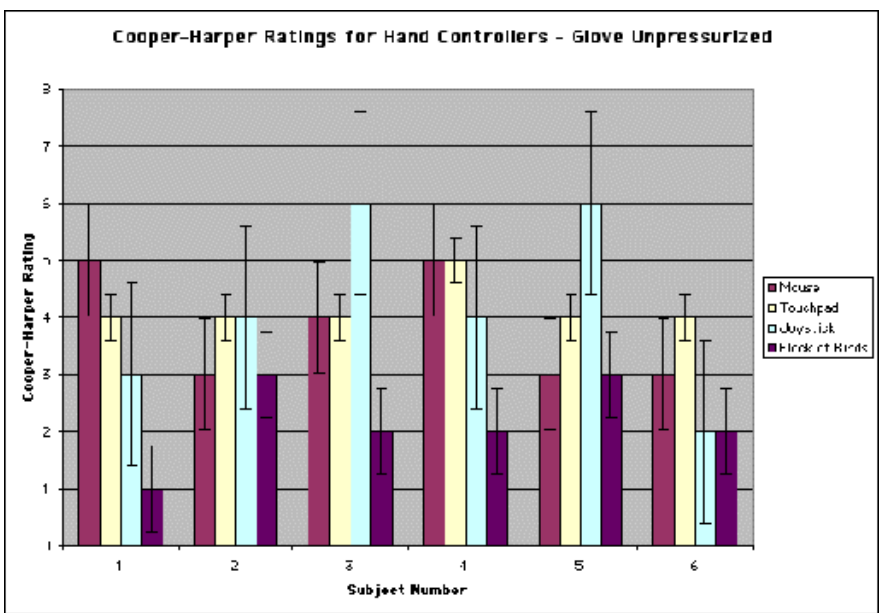

Figure 7: Modified Cooper-Harper Ratings For Hand Controllers Under Unpressurized Glove Conditions

Here, three subjects agree that the magnetic motion sensor controller is best, while two have it tied for first with the mouse and a third with the joystick. The performance of the mouse has decreased from the barehanded, only being ranked as the best with two subjects.

All of the ratings have increased from the barehanded ratings, indicating the increased difficulty with the glove.

The pressurized glove ratings can be seen in Figure 8 .

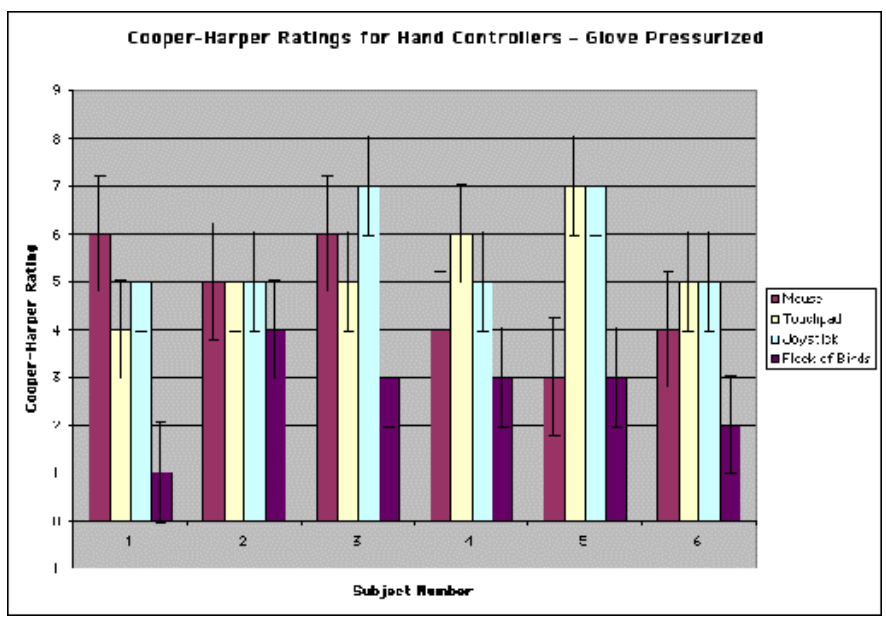

Figure 8: Modified Cooper-Harper Ratings For Hand Controllers Under Pressurized Glove Conditions

In this case, there is a consensus between all subjects that the magnetic motion sensor controller has performed (or tied in one case) the best. The other three hand 
controllers are subject specific, and there is no clear trend.

\section{CONCLUSION}

Overall, the results indicated that the magnetic motion sensor magnetic position controller had the best mean error. The majority of subjects also preferred using this hand controller. This hand controller, however, requires the use of a transmitting antenna and a processing station that may not be practical for all applications. The way in which it functioned for this experiment, using the movement of the arm and shoulder to control an object, could still be used in designing a new type of hand controller.

Several issues were discovered during data collection. Key among them was that the current joystick was not well suited to this experiment, due to resolution problems. This created a deadband at the center of the joystick. Also, since the controller was designed for barehanded use, its shape is not easy to grasp with pressurized gloves. Some subjects used a different method of manipulating the joystick than they did barehanded. This led to some of the varying CooperHarper ratings seen above.

Another issue was that the simulation had an inherent initial error associated with the task. In future work with this software, it would be recommended that a method be developed to force the hand controller and simulation to start at the same initial value.

The last issue is the fit of the gloves. Ideally, all subjects would either have correctly sized gloves, or a much larger pool of subjects selected by hand size would need to be found. Given the time frame of this experiment, it was thought impractical to try to construct such a pool. However, since there is such a clear gender/hand size effect seen here, hand size needs to be taken into greater account in the subject selection process for future research.

From this experiment, it seems that, for a pressurized glove, the most effective controller would be one that did not require a constant grip and that could be controlled by broad motions of the body, as opposed to small, deliberate ones which become quickly fatiguing.

It would also be useful to choose a controller that did not require the use of a spherical grasp, like the mouse, which had the most pressurized complaints. Some subjects adapted instead to avoid the grip completely, instead using just the tip of the index finger to guide the mouse. This was also another case where the motion of the index finger and forearm was preferred over a repetitive hand motion. The index finger strategy worked well with the mouse, but unfortunately did not perform as well with the touchpad. (This was due to the size of the surface of the touchpad in proportion to the glove fingertip size.)
In the future, it would be advantageous to compare a joystick with a redesigned grip, a pressure sensitive touchpad with a larger surface area, and a magnetic position sensor. Once a more definitive gloved hand controller baseline is established, a driving simulation could be used to evaluate these hand controllers for use with robotic assistants.

\section{ACKNOWLEDGMENTS}

This research was developed as a graduate student project under discretionary funding at the University of Maryland Space Systems Laboratory; a part of the Aerospace Engineering Department and the A. James Clark School of Engineering.

The authors would like to thank the following people: Joe Graves for his help and for the use of his graphical software, World Modeler; Steven Weisman, Stephen Roderick, and Jean-Marc Henriette for computer support; Dr. Corde Lane for his comments on human-machine interaction; and last, but certainly not least, the six test subjects that generously donated their time to this project.

Further thanks also go to NASA Goddard Spaceflight Center for their loan of the partial pressure glovebox to the Space Systems Laboratory and ILC Dover, Inc. for their loan of a spacesuit glove.

\section{CONTACT}

For more information on this project, please contact Lauren Shook (shookl@ilcdover.com) or Dr. David Akin (dakin@ssl.umd.edu) at the Space Systems Laboratory, Bldg 382, University of Maryland, College Park, 20742.

\section{REFERENCES}

1. Roberts, Brian, Shook, Lauren, Hossaini, Lisa, Cohen, Robert, "Analysis of Three-Dimensional Roller Performance in a Micro-g Environment," 1999 Shuttle Small Payloads Project Symposium Proceedings, NASA-CP-1999-209476, 13-15 September 1999 (SSL Document Number 99010).

2. Bradley, James V., "Effect of Gloves on Control Operation Time", Journal of Human Factors, 11, 13-20, 1969.

3. Bradley, James V., "Glove Characteristics Influencing Control Manipulability", Journal of Human Factors, 11, 21-36, 1969.

4. Sorenson, E., Sanner, R., Howard, R., Akin, D., Lingo, B., Cadogan, D., "Development of a Power-Assisted Space Suit Glove Joint", 27th International Conference on Environmental Systems, Lake Tahoe, July 1997. 
5. Henshaw, Glen et al. Design of an Astronaut Assistant Rover for Martian Surface Exploration, ENAE 788U Planetary Surface Robotics Final Design Project, SSL Document Number 98-007, December 1998.

6. Trevino, Robert C., Joseph J. Kosmo, and Amy Ross, "First Astronaut-Rover Interaction Field Test", 30th International Conference on Environmental Systems (ICES) and 7th European Symposium on Space Environmental Control Systems\}, July 2000.

7. Guyer, Tracy L. and Edward Hodgson. "Ready to Wear: Hamilton Sundstrand's Mars Suit" Via Satellite's Launchspace, Volume 5, Number 1, February 1, 2000.

8. Trevino, Robert C., Joseph J. Kosmo, and Amy Ross, "First Astronaut-Rover Interaction Field Test", 30th International Conference on Environmental Systems (ICES) and 7th European Symposium on Space Environmental Control Systems\}, July 2000.

9. Mackenzie, Christine and Thea Iberall, The Grasping Hand, Advances in Psychology 104, Elsevier Science, New York, 1994.

10. Mackenzie, Christine and Thea Iberall, The Grasping Hand, Advances in Psychology 104, Elsevier Science, New York, 1994.

11. Mackenzie, Christine and Thea Iberall, The Grasping Hand, Advances in Psychology 104, Elsevier Science, New York, 1994.

12. Middle Eastern Technical University, ME533 Lecture Notes, Input Devices for CAD Drawing, Touchpad\}, httplverb+://+euclid.ii.metu.edu.trlverb+/ +me53 3lverb+/+demolverb+/+Lecturelverb+_+Noteslve $\mathrm{rb}+/+$ Section1/verb+_1/+||touchpad.htm

13. NASA EVA Research and Technology Roadmap, September 1999.

14. Ascension Technologies Download Package, http://www.acension.com

15. Proctor, Robert W. and Addie Dutta, Skill Acquisition and Human Performance, Sage Publications, Thousand Oaks, 1995.
16. Sheridan, Thomas B., Ferrell, William R., ManMachine Systems: Information, Control, and Decision Models of Human Performance, The MIT Press, Cambridge, MA, 1974.

17. Sheridan, Thomas B., Ferrell, William R., ManMachine Systems: Information, Control, and Decision Models of Human Performance, The MIT Press, Cambridge, MA, 1974.

18. Sheridan, Thomas B., Ferrell, William R., ManMachine Systems: Information, Control, and Decision Models of Human Performance, The MIT Press, Cambridge, MA, 1974.

19. Shook, Lauren. Evaluation of Candidate Hand Controllers for Use by a Space Suit Gloved Subject. Master of Science Thesis, University of Maryland, June 2001.

20. Bishu, Ram R., Klute, Glenn, Investigation of the Effects of Extravehicular Activity (EVA) Gloves on Performance, NASA Technical Paper, NASA TP-3401, 1993.

21. Shook, Lauren. Evaluation of Candidate Hand Controllers for Use by a Space Suit Gloved Subject. Master of Science Thesis, University of Maryland, June 2001.

\section{DEFINITIONS, ACRONYMS, ABBREVIATIONS}

ANOVA - Analysis of Variance

DOF - Degree of Freedom

EVA - Extravehicular Activity

EMU - Extravehicular Mobility Unit

ISS - International Space Station

NASA -National Aeronautics and Space Administration

SAS- Statistical Analysis Software

TMG - Thermal Micrometeorite Garment 